\title{
Valuing Individuals' Preferences and Health Choices of Physical Exercise
}

\author{
Emmanuel Aboagye (D)
}

Received: December 23, 2016/Published online: March 4, 2017

(c) The Author(s) 2017. This article is published with open access at Springerlink.com

\begin{abstract}
The efficacy of physical exercise for the prevention and treatment of non-specific low back pain (LBP) is well documented, but little is known about how individuals value specific components of physical exercise, such as the type and design or the intensity and frequency of exercise. Other factors that influence individual differences in health choices and adherence are associated with individuals' attitudes toward and likelihood of performing recommended exercise regimens. Current evidence shows that efficacy is similar among exercise interventions, but their features vary widely. Thus it may be difficult for clinicians to discriminate between available options in clinical practice. Considering the many challenges in determining the form of exercise best suited to the individual patient, this commentary discusses some of the practical methods that could be used to elicit individual preference for recommended health care interventions. Such
\end{abstract}

Enhanced content To view enhanced content for this article go to http://www.medengine.com/Redeem/ 5197F0602F2501B1.

E. Aboagye $(\bowtie)$

Department of Environmental Medicine, Unit of Intervention and Implementation Research for

Worker Health, Karolinska Institute, Stockholm, Sweden

e-mail: emmanuel.aboagye@ki.se methods have the advantage of providing more information for health care decision making, particularly with regard to exercise interventions for LBP. This commentary also advocates for the use of patient preference in health care decisions.

Keywords: Discrete choice experiment; Low back pain; Physical exercise; Stated preference

\section{COMMENTARY}

In economic decision making, the practice of determining who gets what and who pays for what in light of resource constraints and efficiency requirements is considered the rule rather than the exception. From a resource allocation point of view, a country's health care systems should provide the greatest value for the money, given that resources are limited. In recent years, high-income economies have increasingly expended enormous resources in providing health care, yet there is widespread dissatisfaction with what such expenditures have achieved in terms of health outcomes and whether those resources are allocated appropriately. Government pockets have become increasingly shallow, and health care resources in the form of labor and capital are dwindling as pressure mounts.

Health care decision makers are entrusted with the task of determining the value of health 
benefits, and even individual health choices, in order to recommend effective and efficient health interventions that taxpayer money can support. Over the years, cost-effectiveness analysis (CEA) and cost-utility analysis (CUA) have become common methods for measuring individual values of intermediate and final health outcomes such as biomedical markers, the quantity and quality of life gained (i.e., quality-adjusted life-year [QALY]), or the avoidance of an adverse event (e.g., death) through the use of health care interventions [1]. Priorities are set based on these and other criteria, including the cost of the intervention. However, allocation of health care resources for different diagnoses largely relies on these methods using comparative analysis of at least two alternative interventions to support their recommendation.

In intervention research, there are myriad indications of mediating factors impacting both proximal and distal intervention outcomes. Mediators such as individual components of the intervention and patient-related factors (e.g., lifestyle, attitudes, preferences) are likely to have considerable influence on acceptance and compliance rates, and consequently on health outcomes [2]. In order for interventions targeting behavioral changes to have any positive effect, a patient-centered model of health care is widely advocated. Domains of the patient-centered model such as understanding and communicating with the patient, exploring the experience of illness, and enhancing health and the patient-doctor relationship can also mediate health outcomes [3]. From these domains, preferences according to factors such as age, socioeconomic status, level of illness severity and patient anxiety, and the familiarity of the doctor with the patient may bring important differences in health outcomes. This implies that the preference for certain features of an intervention is fundamental to supporting its recommendation, particularly for patients with a strong preference $[4,5]$. Research on individuals' preferences for health care interventions in general, however, is scarce.

For the purposes of this commentary, let us select a prevalent diagnosis in public health care in most high-income economies today, low back pain (LBP) [6]. LBP affects $60-80 \%$ of the population at some point in their lifetime [7]. The condition is characterized by a chain of episodes, i.e., recurrent pain [8]. Aside from the burden of LBP on those affected, it contributes substantially to an economic burden in terms of productivity loss in high-income countries [9]. Indeed, a major portion of the increasing absenteeism due to illness in such economies has been attributed to LBP [10]. The number of individuals with LBP is expected to increase substantially over the coming decades as the population in these countries ages [11]. Therefore, interventions to improve back health are critical.

Various forms of exercise interventions have traditionally been used to manage LBP, some of which have been evaluated using patient perceptions of their value in a CEA/CUA $[12,13]$. There is evidence of the effectiveness of exercise interventions for LBP pointing to benefits such as minimizing the risk of recurrent back pain [14]. Some questions that have been partially addressed include the types of programs to provide and the appropriate level at which to provide them. The current evidence shows that efficacy is similar among exercise interventions $[15,16]$, but their components vary widely. Thus an interesting question that perhaps should be considered is what individual patients prefer among the various exercises for the prevention and treatment of LBP. The answer to this question may also suggest ways to promote individual participation in and adherence to exercise interventions recommended for them.

This way of thinking may have some inherent flaws. Particularly for physical exercise interventions, standard techniques for understanding individual preferences may be inadequate. It may not be enough to simply ask patients to choose their preferred form of exercise for preventing or treating their back pain. Given the heterogeneity among individuals as well as unknown factors, certain irrational choices can be expected. A different approach may be needed, not only for assessing patient health choices, but also for providing informa- 
tion about the appropriate level of care in such diverse decision contexts. Furthermore, in order to engage individuals in a particular physical exercise, details of what constitutes the exercise program should be described as components of a set.

Views also differ on the specific features of physical exercise most valued by individuals, such as whether it is supervised, whether it involves individual or group sessions, or what the optimal exercise intensity should be [17]. Through behavioral research, there have been proposals to explain the relative importance of characteristics of interventions to patients to better understand patients' health-related choices as well as the possibility of increased uptake by eliminating undesired attributes and focusing more on the desired attributes of an intervention [18]. In order to ensure that health care resources are used efficiently, choices can be provided and preferences assessed such that the physical exercise interventions that individuals with back problems prefer and are willing to engage in are those that are designed and paid for. The question of who will pay is a matter beyond the scope of this commentary.

Preference and choice are important concepts in the experimental analysis of health behavior. Preference is measured as a pattern of choosing or responding when presented with two or more alternatives, and often requires a choice to be made [19]. The fundamental question regarding preference and choice is what causes an individual to behave in one way or the other when presented with two or more alternatives at any given time.

Considering the many challenges in understanding what patients would prefer in the form of exercise, this commentary focuses on some of the practical applications that could be used to elicit individual preferences to enhance the effectiveness of health care intervention recommendations. The methods discussed have the advantage of providing more information for health care decision making, particularly with regard to exercise interventions for LBP. This commentary also advocates for the use of patient preference in health care decision making.
The notion that an individual's perceived value of goods and services is dependent on the nature of the goods or service is well investigated in decision theory [20]. In health care decision making, there are two techniques that can be used to study how individuals place importance on health care services and their associated benefits. One technique assumes that the individuals' choices reveal their preference. This is an indirect means of exploring individual values of health benefits, known as the "revealed preference" (RP) technique [21]. As an example, one study that used this method examined individuals' preferences for flexibility and comfort as well as their environmental preferences in order to gain an understanding of their choice of travel mode [22]. A second technique involves asking individuals to state their preferences by presenting them with options in hypothetical scenarios. This is known as the "stated preference" (SP) technique, and it has seen increased interest with regard to valuing health care choices [23]. For example, the SP technique was used in a study examining LBP patients' preferences among interventions that differed by type but had similar outcomes, such as acupuncture or low frequency-infrared therapy [24]. The two most widely used SP techniques for estimating the value of health choices are the contingent valuation method (CVM) and the discrete choice experiment (DCE). The strengths and weaknesses of these techniques, and how the SP techniques in particular have been used in research on physical exercise in different populations, are discussed below.

The underlying assumption in using an RP technique is that individual preferences are revealed by the utility derived from the use of the health care intervention. The SP technique can provide much more detail than simply what is revealed by the use of the health care intervention being examined. Thus, if one thinks of the goal of a study in terms of answering questions about exercise interventions as a whole (e.g., how much individuals are willing to pay for a recommended exercise program), a CVM study may be appropriate. If the aim is to study the importance of the different characteristics of the exercise intervention, DCEs may be more 
suitable [25]. This is not to say that both the RP and SP techniques may not be suitable for examining patients' health choices and possible benefits of exercise interventions for LBP. The use of both techniques is feasible.

In health care, the fundamental reasons for choosing the SP over the RP technique are that there are aspects of individuals' choices that tend not to be fully rational, and these interventions can be subsidized via health insurance coverage. This is the case in many high-income economies. And if physical exercise is in fact subsidized by taxpayer-funded health insurance or paid for by a third party, individuals' choices may not necessarily reveal their preferences or their perceptions of the value of health benefits when data is gathered using an RP approach. Even with that payment structure, failure to take into account the patient's perspective (i.e., if the health intervention is not based on the patient's preference) could lead to misallocation of scarce resources when subsidized health insurance is involved and patients simply choose not to do what is recommended to them.

There are also other strengths and weaknesses in SP techniques. In relation to exercise intervention research in particular, non-economic rationales may introduce weaknesses, because individuals may say they will do one thing (SP) and actually do another (RP). An additional aspect of the non-economic rationale is that an individual's choice of exercise intervention could be influenced by the relationship between the clinician (including physiotherapists) and the individual [26]. In the provision of health care, the clinician is presumably better informed than the patient. This is an information asymmetry problem, which is a notorious issue in health care delivery [27]. Of course, consumers can also be influenced by advertising, by which they may become better informed or, alternatively, they may be deceived, and then they may become discouraged and abandon attempts at other forms of exercise. Supposing that the clinician is better informed than the patient, it is obvious where the information imbalance exists. Then again, there is also a biological factor to consider, which is the physical capacity of the individual. An individual may not be comfortable with a certain activity, thus reducing the likelihood of their participation in the activity, their adherence to exercise recommendations, and their prospects for recovery. Therefore, individual preference becomes relevant for many reasons, such as time and capacity constraints, that express the ways in which individuals can improve their health state.

It should be added that the act of choosing in itself can be a tedious task for patients; thus practitioners are tempted to involve individuals in treatment decision-making processes as little as possible. However, at a certain point it becomes reasonable to assume that relying solely on the treatment or the clinician in determining exercise recommendations for the prevention and treatment of LBP cannot ensure improved health outcomes. There are also other factors, particularly patient preferences, that are fundamental to obtaining the desired outcomes. Measuring this preference concept, so to say, is an undertaking that should be given considerable thought and must be better understood.

Last but not least, another strength of the SP technique is that it allows large quantities of relevant data to be collected at a moderate cost. To a large extent, DCEs provide more information than a CVM experiment [26]. Based on hypothetical choices, which can be specified a priori using specific experimental designs, identification of the relative importance of the characteristics of an intervention can be estimated in a straightforward manner [18]. This is in contrast to RP data, which cannot be controlled a priori, and the same is implied for model specification.

A few studies have used SP techniques to value individual health choices of physical exercise interventions among different sample groups. In one study, a CVM was used to value willingness to pay with the introduction of physical activity on prescription in the Swedish health sector [28]. The impact of financially incentivized physical exercise programs among older people (to enhance mobility and prevent falls), elicited using a DCE approach, showed a positive influence on physical activity behavior $[29,30]$. Some of the components of physical 
exercise used to establish choices for individuals expressing their preferences in the aforementioned research include the frequency of exercise sessions, travel distance, program fees, and financial incentives. These are some of the key features of a physical exercise regimen that have been shown to improve adherence for adults with non-specific LBP [31]. Other characteristics of exercise associated with increased adherence-and which warrant further research-include the type of activity, intensity, and design (i.e., group versus individual physical activity, with or without trainer supervision).

The application of SP techniques, however, and especially DCEs, is still relatively rare in samples of LBP patients where physical exercise interventions have been shown to be effective. In future research, DCEs can be used to estimate the effects of components of exercise interventions, including monetary incentives, which are valued by individuals with non-specific LBP. Such individual-based values of exercise interventions can guide the development of new approaches for LBP patients, enhancing intervention uptake as well as health outcomes [2]. There may be situations where the two SP techniques can be combined to enhance data quality in measuring outcomes of welfare interest and health care value.

In occupational health economics, the use of DCE techniques is limited. In order to promote employee back health, DCEs can be used to value preference for workplace physical exercise interventions. An advantage during the testing of such interventions will be to engage both employers and employees and to study potentially desirable physical activity characteristics before developing the profiles for the choice experiment. The employer and the researcher could discuss the best- and worst-case scenarios based on job characteristics in order to choose characteristics that are adaptable to the work setting and to create choice for employees [32]. Here, preference values at both the individual and aggregated group levels can be examined simultaneously. Such an experiment that involves both employers and employees may facilitate faster implementation of the workplace exercise program, because the outcomes are more likely to be adaptable to work situations.
DCEs can potentially contribute to the measurement of desirable characteristics of exercise interventions for individuals with LBP. The fundamental principles and steps in carrying out DCEs have been covered in the literature $[33,34]$. The quality of DCEs can be improved by following a checklist that includes conceptualizing the choice process, determining appropriate levels for attributes, choosing an experimental design, designing the questionnaires, pilot testing, sampling and determining sample size, collecting data, coding data, and performing econometric analysis, interpretation, welfare, and policy analysis [35]. A report on good research practices by the International Society for Pharmacoeconomics and Outcomes Research (ISPOR) Conjoint Analysis Task Force further outlines detailed design requirements and summarizes a number of approaches for constructing choice experiments [36]. The report can assist researchers in evaluating alternative approaches for experimental designs-the most important aspect of conducting a successful DCE. Prior to designing a study using an SP technique, the views of expert researchers on the experimental design are solicited to avoid unforeseen challenges in the analysis of data.

In summary, individuals place significant value on features that differentiate exercise interventions for the prevention and treatment of non-specific LBP. SP techniques can be used to estimate the effects of specific components of exercise interventions on an individual's health choices. The technique may be much more useful when studying interventions for modifiable behavior or health risk-for instance, physical exercise for prevention and treatment of LBP. In order to capture the value of exercise programs from a patient perspective, preferences for treatment features should be considered in evaluating such programs. In practice, health providers and employers may take into account the relative importance of specific desirable characteristics in order to align health care decision making and implementation with individual preferences. This could improve the effectiveness of exercise programs through enhanced patient satisfaction and adherence in the prevention and treatment of non-specific 
LBP. Studies seeking to test the efficacy of interventions can also adopt the views of at-risk groups on what they believe are important components of an intervention, so that these features can be incorporated into the design of the intervention prior to investigating its efficacy.

\section{ACKNOWLEDGEMENTS}

No funding or sponsorship was received for this study or publication of this article. All named authors meet the International Committee of Medical Journal Editors (ICMJE) criteria for authorship for this manuscript, take responsibility for the integrity of the work as a whole, and have given final approval for the version to be published. The author is grateful to Professor Irene Jensen and Associate Professor Iben Axén for their valuable comments on this commentary.

Disclosures. Emmanuel Aboagye has nothing to disclose.

Compliance with Ethics Guidelines. This article is based on previously conducted studies and does not involve any new studies of human or animal subjects performed by the author.

Open Access. This article is distributed under the terms of the Creative Commons Attribution-NonCommercial 4.0 International License (http://creativecommons.org/licenses/by-nc/4. $0 /$ ), which permits any noncommercial use, distribution, and reproduction in any medium, provided you give appropriate credit to the original author(s) and the source, provide a link to the Creative Commons license, and indicate if changes were made.

\section{REFERENCES}

1. Drummond $\mathrm{M}$, et al. Methods for the economic evaluation of health care programmes. Oxford: Oxford University Press; 2005.
2. Klaber Moffett JA, et al. Randomised trial of a brief physiotherapy intervention compared with usual physiotherapy for neck pain patients: outcomes and patients' preference. BMJ. 2005;330(7482):75.

3. Little $P$, et al. Preferences of patients for patient centred approach to consultation in primary care: observational study. BMJ. 2001;322(7284):468-72.

4. George SZ, Robinson ME. Preference, expectation, and satisfaction in a clinical trial of behavioral interventions for acute and sub-acute low back pain. J Pain. 2010;11(11):1074-82.

5. Preference Collaborative Review G. Patients' preferences within randomised trials: systematic review and patient level meta-analysis. BMJ. 2008;337:a1864.

6. Hoy D, et al. The epidemiology of low back pain. Best Pract Res Clin Rheumatol. 2010;24(6):769-81.

7. Hoy D, et al. The global burden of low back pain: estimates from the Global Burden of Disease 2010 study. Ann Rheum Dis. 2014;73(6):968-74.

8. Axen I, Leboeuf-Yde C. Trajectories of low back pain. Best Pract Res Clin Rheumatol. 2013;27(5):601-12.

9. Smith E, et al. The global burden of other musculoskeletal disorders: estimates from the Global Burden of Disease 2010 study. Ann Rheum Dis. 2014;73(8):1462-9.

10. Coggon D, et al. International variation in absence from work attributed to musculoskeletal illness: findings from the CUPID study. Occup Environ Med. 2013;70(8):575-84.

11. Hoy D, et al. A systematic review of the global prevalence of low back pain. Arthritis Rheum. 2012;64(6):2028-37.

12. Aboagye E, et al. Cost-effectiveness of early interventions for non-specific low back pain: a randomized controlled study investigating medical yoga, exercise therapy and self-care advice. J Rehabil Med. 2015;47(2):167-73.

13. Chuang LH, et al. A pragmatic multicentered randomized controlled trial of yoga for chronic low back pain: economic evaluation. Spine (Phila Pa 1976). 2012;37(18):1593-601.

14. Hayden JA, et al. Meta-analysis: exercise therapy for nonspecific low back pain. Ann Intern Med. 2005;142(9):765-75.

15. Hayden JA, et al. Exercise therapy for treatment of non-specific low back pain. Cochrane Database Syst 
Rev. 2005;(3):CD000335. doi:10.1002/14651858. CD000335.pub2.

16. Tveito TH, Hysing M, Eriksen HR. Low back pain interventions at the workplace: a systematic literature review. Occup Med Oxford. 2004;54(1):3-13.

17. Henchoz Y, Kai-Lik So A. Exercise and nonspecific low back pain: a literature review. Joint Bone Spine. 2008;75(5):533-9.

18. Louviere JJ, Lancsar E. Choice experiments in health: the good, the bad, the ugly and toward a brighter future. Health Econ Policy Law. 2009;4(Pt 4):527-46.

19. Martin TL, et al. On choice, preference, and preference for choice. Behav Anal Today. 2006; 7(2):234-41.

20. Lancaster K. A new approach to consumer theory. J Polit Econ. 1996;74(2):132-57.

21. Adamowicz W, Louviere J, Williams M. Combining revealed and stated preference methods for valuing environmental amenities. J Environ Econ Manag. 1994;26(3):271-92.

22. Vredin Johansson M, Heldt T, Johansson P. The effects of attitudes and personality traits on mode choice. Transp Res Part A Policy Pract. 2006;40(6):507-25.

23. Louviere J, Hensher DA, Swait JD. Stated choice methods: analysis and application. Cambridge: Cambridge University Press; 2000.

24. Chen LC, et al. Acupuncture or low frequency infrared treatment for low back pain in Chinese patients: a discrete choice experiment. PLoS One. 2015;10(5):e0126912.

25. Amaya-Amaya M, Gerard K, Ryan M. Discrete choice experiments in a nutshell. In: Ryan M, Gerard $\mathrm{K}$, Amaya-Amaya $\mathrm{M}$, editors. Using discrete choice experiments to value health and health care. Dordrecht: Springer; 2008.

26. Gerard K, Ryan M, Amaya-Amaya M. Introduction. In: Ryan M, Gerard K, Amaya-Amaya M, editors.
Using discrete choice experiments to value health and health care. Dordrecht: Springer; 2008.

27. Shmanske S. Information asymmetries in health services the market can cope. Indep Rev. 1996;1(2):191-200.

28. Rome A, et al. Willingness to pay for health improvements of physical activity on prescription. Scand J Public Health. 2010;38(2):151-9.

29. Franco MR, et al. Eliciting older people's preferences for exercise programs: a best-worst scaling choice experiment. J Physiother. 2015;61(1):34-41.

30. Farooqui MA, et al. Effects of financial incentives on motivating physical activity among older adults: results from a discrete choice experiment. BMC Public Health. 2014;14:141.

31. Jordan JL, et al. Interventions to improve adherence to exercise for chronic musculoskeletal pain in adults. Cochrane Database Syst Rev. 2010;(1):CD005956. doi:10.1002/14651858.CD005956.pub2.

32. Muhlbacher AC, et al. Experimental measurement of preferences in health care using best-worst scaling (BWS): theoretical and statistical issues. Health Econ Rev. 2015;6(1):5.

33. Lancsar E, Louviere J. Conducting discrete choice experiments to inform healthcare decision making: a user's guide. Pharmacoeconomics. 2008;26(8):661-77.

34. Ryan M, Gerard K. Using discrete choice experiments to value health care programmes: current practice and future research reflections. Appl Health Econ Health Policy. 2003;2(1):55-64.

35. Bridges JF, et al. Conjoint analysis applications in health-a checklist: a report of the ISPOR Good Research Practices for Conjoint Analysis Task Force. Value Health. 2011;14(4):403-13.

36. Reed Johnson F, et al. Constructing experimental designs for discrete-choice experiments: report of the ISPOR Conjoint Analysis Experimental Design Good Research Practices Task Force. Value Health. 2013;16(1):3-13. 